ISSN 1991- 8690

website:http:// jsci.utq.edu.iq

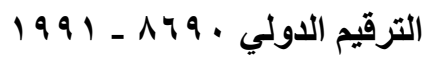

Email: utjsci@utq.edu.iq

\title{
PRE-ALEXANDROFF SPACE
}

\author{
Ayed .E. Hashoosh Al-Badry \\ University of Thi-Qar College of Education of pure sciences \\ Dept. of Mathematics . \\ Email:Ayed197991@yahoo.com
}

\begin{abstract}
In this paper we introduce a new definition of the topological space weaker of Alexandroff space, namely pre-Alexandroff space. These spaces are which arbitrary intersection of an open set is a pre-open set. In addition to give a new definition of minimal pre-open sets and investigate about some of its properties, also we get some theorems and result related to this space.

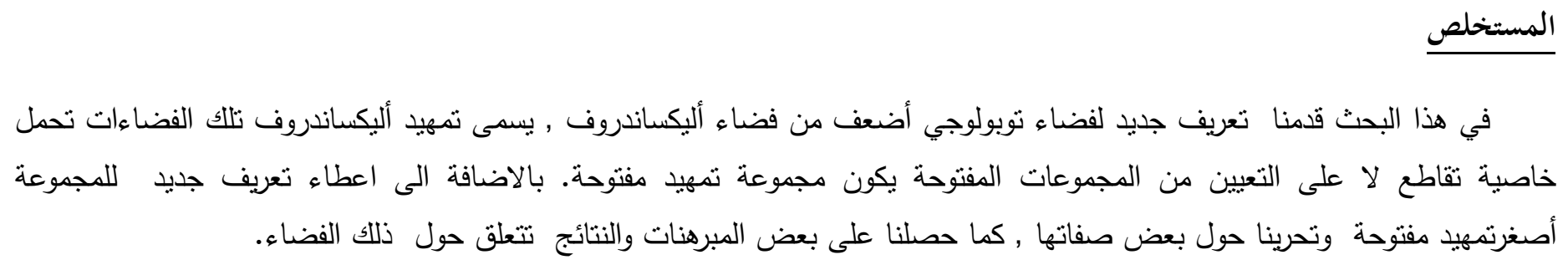

\section{Introduction}

$\tau_{0}$-Alexandroff spaces have been the subject of many research papers recently, see [1], [2], [4], [5], [7], [9], [11] and [12]. This space is used in digital topology applications and digital image analysis. Other approaches to digital topology, which lead to locally finite spaces are for example, the models based on cell complex developed by Kovalevsky [7] and Rosenfeld [11,12] These latter papers apply discrete topological models in theoretical physics as well.

An Alexandroff space is a topological space in which arbitrary intersection of open sets is open [1]. This equivalent to saying that every point of $\mathrm{X}$ has a minimal open set. The class of Alexandroff spaces includes all finite topological spaces. In fact, each finite topological space is locally finite, and each locally finite is Alexandroff .This space was studied first time in 1937 by P. Alexandroff under the name of discrete Raume (discrete space). The name is not valid now, since discrete space is a space where the singletons are open.

In this paper, we give definition of new topological space, namely a pre-Alexandroff space in which arbitrary intersection of open sets is pre-open set.This space is not in general is Alexandroff space. 
The concepts of pre-open set and pre-open function in topological spaces was introduced in 1982 [8] .These concepts were also considered in [6] and [13].

Moreover, We got a series of theorems and important results, as well as that we have minimal pre-open and we proved it the base for this space and denoted it by MP (x). Among the new results obtained in this research. The property pre-A-space hereditary property, also the continuous image of pre-A-space is not necessarily pre-A-space. Finally, we study the Hausdoroff, Product and Quotient topology in a pre-A-space. The aim of this paper is to continue the discussion new classes are weakly of Alexandroff spaces [3] .

\section{Preliminaries}

In this section, we recall the basic definitions and facts needed in this paper.

\subsection{Definition [1]}

An Alexandroff Space is a topological space in which arbitrary intersection of open sets is open.

\subsection{Definitions [8]}

1) Let $X$ be a topological space and $A \subseteq X$. A is called pre-open in $X$ if and only if $A \subseteq$ int $(\operatorname{cl}(A)$. $A$ is called pre-closed if and only if the complement of $\mathrm{A}$ is pre-open.

It is clear that every open set is pre-open and every closed set is pre-closed, but the converse is not true in general. The intersection of two pre-open sets is not, in general pre-open, but the intersection of pre-open set with open set is pre-open. Also the union of any pre -open sets is pre-open .

2) Let $f$ be a function from a space $X$ into a space $Y$, then $f$ is called pre-open function if $f(U)$ is preopen in $\mathrm{Y}$ for each open set in $\mathrm{X}$.

\subsection{Definition [5]}

A topological space $(X, \tau)$ is called locally finite if each element $\mathrm{x}$ of $\mathrm{X}$ is contained in a finite open set and a finite closed set.

\subsection{Theorem [5]}

(i) Each finite space is locally finite space .

(ii) Each locally finite space is Alexandroff space .

\subsection{Definition [10]}

A space $(X, \tau)$ is called a sub maximal if each dense subset is an open set.

$\underline{\text { 2.6 Theorem }[10]}$

Let $(X, \tau)$ be a topological space, then the following are equivalent:

1. $\mathrm{X}$ is a sub maximal.

2 . Every a pre-open set is an open.

\section{Pre-Alexandroff Spaces}

In this section, we give some important results and theorems for pre- Alexandroff Space. 


\subsection{Definition:}

A pre-Alexandroff space is a topological space in which arbitrary intersection of an open sets is a pre-open , and denoted by (pre-A-space) .

This property does not hold in all topological spaces, for example in the usual topology on $\mathrm{R}$ $\bigcap_{n \in N}\left(-\frac{1}{n}, \frac{1}{n}\right)=\{0\} \quad$ which is not pre-open in $\mathrm{R}$. Hence $\left(\mathrm{R}, \mathrm{T}_{\mathrm{u}}\right)$ is not a pre-A-space .

\subsection{Theorem:-}

Let $\mathrm{X}$ be a topological space contains a point $\{\mathrm{k}\}$ such that $\{\mathrm{k}\}$ is open and dense, then $\mathrm{X}$ is a pre-A-space . Proof : - Suppose that $\left\{\alpha_{\mathrm{i}}:{ }_{\mathrm{i}} \in \mathrm{I}\right\}$ be a collection of open sets, and $\alpha$ be it is an intersection .

Suppose $\alpha$ is not pre-open set, it means that $\alpha \not \subset \operatorname{int}(\operatorname{cl}(\alpha)$. But $\alpha \subseteq\{\mathrm{k}\}$, because $\alpha$ is an intersection of all open set and $\{\mathrm{k}\}$ open set. Hence $\operatorname{int}(\operatorname{cl}(\alpha))=\operatorname{int}(\operatorname{cl}\{k\}=X$, therefore $\alpha \not \subset X$, that is a contradiction . Thus $X$ is a pre-A-space .

\subsection{Remark:-}

1) Every an Alexandroff space is a pre-A-space.

2) Every a finite space is a pre-A-space.

3) Every a locally finite space is a pre-A-space.

Proof :- Are obvious, since open set is pre-open. But the convergence of the above Remark (3.3) is not true, the following example showing that not pre-A-space is an Alexandroff space.

\subsection{Example :-}

Let $\mathrm{X}$ be the real line with the topology $\tau=\{\varphi, X,\{0\}\} \cup\{G \subseteq X: 0 \in \mathrm{G}, \mathrm{XIG}$ finite $\}$, clearly $\{0\}$ is open and dense for a topological space $\mathrm{X}$, hence $\mathrm{X}$ is a pre-A-space by Theorem (3.2). On the other hand the intersection of all open sets $X \backslash\{x\}$ such that $x$ is irrational, is not open. Therefore $X$ is not Alexandroff space.

\subsection{Theorem:-}

Every $\tau_{1}$-pre-A- space is an Alexandroff .

Proof :- Let $\mathrm{X}$ be $\tau_{1^{-}}$space . Let $\mathrm{x} \in \mathrm{X}$ and $\Omega=\cap \mathrm{U}$, where $\mathrm{U}$ is arbitrary open set contains $\mathrm{x}$. There are two possibilities, either $\Omega$ contains a single point $\mathrm{x}$ or more point .

Now if $\Omega$ contains more than one element. Let $\mathrm{y} \in \Omega$ such that $\mathrm{x} \neq \mathrm{y}$, since $\{\mathrm{y}\}$ is closed then $\{\mathrm{y}\}^{\mathrm{c}}$ is an open set contains $\mathrm{x}$. Hence $\Omega \subseteq\{\mathrm{y}\}^{\mathrm{c}}$ but $\mathrm{y} \in \Omega \subseteq\{\mathrm{y}\}^{\mathrm{c}}$. This is a contradiction, hence $\Omega$ contains one element $\{x\}$. Therefore $\{x\}$ is pre-open set because $X$ is a pre-A-space, but $\{x\}$ is closed, therefore $\{x\}$ is an open set, hence $\mathrm{X}$ is an Alexandroff space .

\subsection{Remark [4]}

Every $\tau_{1}$-Alexandroff is a discrete space .

\section{$\underline{\text { 3.7 Corollary }}$}

Every $\tau_{1}$-pre-A-space is a discrete space .

Proof :- By Theorem (3.5) and Remark (3.6 ) 


\subsection{Corollary}

Let $\left(\mathrm{R}, \tau_{\mathrm{c}}\right)$ be co-finite topology on real number then $\left(\mathrm{R}, \tau_{\mathrm{c}}\right)$ is not a pre- $\mathrm{A}-$ space.

Proof : - Suppose to the contrary that $\left(\mathrm{R}, \tau_{\mathrm{c}}\right)$ is a pre-A- space, but $\left(\mathrm{R}, \tau_{\mathrm{c}}\right)$ is a $\tau_{1}$-space, therefore $\left(\mathrm{R}, \tau_{\mathrm{c}}\right)$ is a discrete space by Corollary (3.7) which is a contradiction .

\subsection{Theorem [2]}

If $\beta$ is a collection of subsets of $X$ such that for each $x \in X$, there is a minimal set $m(x) \in \beta$ containing $\mathrm{x}$, then $\beta$ is a basis for a topology on $X$ and $X$ is an Alexandroff space with this topology.

The following corollary is a special case of the above Theorem .

\subsection{Corollary}

Let $X$ be pre-A-space, and $\beta$ is a collection of subsets of $X$ such that for each $\mathrm{x} \in \mathrm{X}$, there is a minimal pre-open set MP $(\mathrm{x}) \in \beta$ containing $\mathrm{x}$, then $\beta$ is a basis for a pre-A-space .

From here on we denoted a minimal pre-open is $\mathrm{MP}(\mathrm{x})$ containing $\mathrm{x}$.

\subsection{Theorem}

Let $\mathrm{X}$ be pre-A-space if and only if $\mathrm{X}$ has $\mathrm{MP}(\mathrm{x})$ for any $\mathrm{x} \in \mathrm{X}$.

Proof: $\Rightarrow$ Suppose $X$ is pre-A-space. Let $\Omega(\mathrm{x})=\{\mathrm{U} \subseteq \mathrm{X}$ : $\mathrm{U}$ is an open set of $\mathrm{x}$.

Now $\beta(x)=\cap U$, for $U \in \Omega$, but $X$ is pre-A-space, therefore $\beta(x)$ is pre-open set of $x$. So $\beta(x)$ is $\operatorname{MP}(x)$, hence $\mathrm{X}$ has $\mathrm{MP}(\mathrm{x})$ for any $\mathrm{x} \in \mathrm{X}$.

$\Leftrightarrow)$ Suppose $X$ has MP(x) for any $x \in X$. Take $A=\bigcap_{a \in \Lambda} U_{a}$ arbitrary intersection of open set .

Now, if $A$ is empty set then $A$ is pre-open set, and we are done. Suppose $A$ has at least one element $x \in A$, then $\mathrm{x} \in \mathrm{U}_{\mathrm{a}}$ for any $\mathrm{a} \in \Lambda$. But $\mathrm{U}_{\mathrm{a}}$ is open set, hence $\mathrm{U}_{\mathrm{a}}$ is pre-open, because every open is pre-open, therefore there exist MP(a) $\subseteq \mathrm{U}_{\mathrm{a}}$ for any a $\in \Lambda$ therefore MP (a) $\subseteq \mathrm{A}$, because MP(a) is minimal pre open set contains $a$.

Now for every $\mathrm{x} \in \mathrm{A}$, there exist $\mathrm{MP}(\mathrm{x})$ containing $\mathrm{x}$ such that $\mathrm{x} \in \operatorname{int}(\mathrm{cl}(\mathrm{MP}(\mathrm{x})) \subseteq \operatorname{int}(\mathrm{cl}(\mathrm{A}))$. That means that $\mathrm{A}$ is a pre-open set. Thus $\mathrm{X}$ is a pre-A-space .

\subsection{Theorem}

Let $\mathrm{Y}$ be a subspace of pre-A-space $\mathrm{X}$, then $\mathrm{Y}$ is pre-A- space .

Proof :- Suppose that $\left\{U_{a}: a \in \Lambda\right\}$ be a collection of open set in $\mathrm{Y}$. Take $\mathrm{A}=\bigcap_{a \in \Lambda} U_{a}$ arbitrary intersection of open set in Y, we shall prove A is pre-open set $A=\bigcap_{a \in \Lambda} U_{a}=\bigcap_{a \in \Lambda}\left(V_{a} \cap Y\right)=Y \cap\left(\cap_{a \in \Lambda} V_{a}\right) \subseteq Y \cap$ $\left(\bigcap_{a \in \Lambda} \operatorname{int}\left(\operatorname{cl}\left(V_{a}\right)\right) \subseteq \operatorname{int}\left(\operatorname{cl}\left(Y\left(\cap_{a \in \Lambda} V_{a}\right)\right)=\operatorname{int}\left(\operatorname{cl}\left(\cap_{a \in \Lambda} U_{a}\right)=\operatorname{int}(\operatorname{cl}(A)\right.\right.\right.$. Therefore $A$ is pre-open set. Thus $\mathrm{Y}$ is pre-A- space .

\subsection{Theorem}

Let $\mathrm{f}: \mathrm{X} \rightarrow \mathrm{Y}$ be a pre-open and continuous function from a pre-A-space $\mathrm{X}$ onto a space $\mathrm{Y}$. Then $\mathrm{Y}$ is also a pre-A-space .

Proof :- Let $x \in X$, then there exist $y \in Y$ such that $f(x)=y$.Then $f(V)$ is pre-open set contains $y$ for every open set $\mathrm{V}$ in $\mathrm{X}$ contains $\mathrm{x}$ because $\mathrm{f}$ is pre-open function .

Now, we take $\mathrm{U}$ is an open set in $\mathrm{Y}$ contains $\mathrm{y}$. Therefore $\mathrm{x} \in \mathrm{f}^{-1}(U)$, but $\mathrm{f}^{-1}(U)$ is open set in $\mathrm{X}$ because $\mathrm{f}$ is a continuous function, hence there exists minimal open set $\mathrm{M}(\mathrm{x}) \subseteq \mathrm{f}^{-1}(U)$.Thus $\mathrm{f}(\mathrm{M}(\mathrm{x})) \subseteq U$, this means $\mathrm{f}(\mathrm{M}(\mathrm{x}))$ is minimal pre-open set contains $\mathrm{f}(\mathrm{x})$ in Y. By Corollary (3.10) hence $\mathrm{Y}$ is a pre-A-space . 


\subsection{Theorem}

Let $\mathrm{X}$ and $\mathrm{Y}$ be two pre-A-space, then $\mathrm{X} \times \mathrm{Y}$ is pre-A- space if and only if both $\mathrm{X}$ and $\mathrm{Y}$ are pre-Aspaces .

Proof :- $\Rightarrow$ ) Let $X \times Y$ has as basis $B=\{U \times V: U$ open in $X, V$ open in $Y\}$, and let $(x, y) \in X \times Y$, then $\operatorname{MP}(\mathrm{x}) \times \operatorname{MP}(\mathrm{y})$ is in $\mathrm{B}$, and the claim is that this is minimal pre-open set in $\mathrm{B}$, contains $(\mathrm{x}, \mathrm{y})$.

If $(\mathrm{x}, \mathrm{y}) \in \mathrm{X} \times \mathrm{Y} \in \mathrm{B}$, then $\mathrm{x} \in \mathrm{X}$ and $\mathrm{y} \in \mathrm{Y}$, so $\mathrm{MP}(\mathrm{x}) \subseteq \mathrm{U}$ and $\mathrm{MP}(\mathrm{y}) \subseteq \mathrm{V}$, hence $\operatorname{MP}(\mathrm{x}) \times \mathrm{MP}(\mathrm{y}) \subseteq \mathrm{U}$ $\times \mathrm{V}$. Therefore $\mathrm{X} \times \mathrm{Y}$ is pre-A- space by Corollary (3.10).

$\Leftarrow$ Let $\mathrm{X} \times \mathrm{Y}$ be pre-A-space, and let $\mathrm{P}_{1}: \mathrm{X} \times \mathrm{Y} \rightarrow \mathrm{X}$ is being the projection of $\mathrm{X} \times \mathrm{Y}$ onto $\mathrm{X}$ defined by $\mathrm{P}(\mathrm{x}, \mathrm{y})=\mathrm{X}$. But the projection function is continuous and pre-open function . Hence by Theorem (3.13) then $\mathrm{X}$ is a pre-A- space and Similar to proof $\mathrm{Y}$ is pre-A- space .

Using induction, if $\mathrm{Xi}, \mathrm{i}=1,2, \ldots, \mathrm{n}$ are pre- A- spaces , then $\prod_{i=1}^{n} X i$ is pre-A-space.

\section{15 Remark:}

Let $\mathrm{f}: \mathrm{X} \rightarrow \mathrm{Y}$ be a continuous function, from $\mathrm{X}$ onto $\mathrm{Y}$ and if $\mathrm{X}$ is a pre-A-space then $\mathrm{Y}$ is not necessarily a pre-A-space.

The following example explains the previous Remark.

\subsection{Example}

Let $\mathrm{f}:\left(\mathrm{N}, \tau_{\mathrm{d}}\right) \rightarrow\left(\mathrm{Q}, \tau_{\mathrm{u}} *\right)$ be onto continuous function . $\left(\mathrm{N}, \tau_{\mathrm{d}}\right)$ is a pre-A-space, but $\left(\mathrm{Q}, \tau_{\mathrm{u}} *\right)$ is not pre-Aspace. Where $\tau_{\mathrm{u}} *$ is the relative topology on $\mathrm{Q}$ as a subspace of $\left(\mathrm{R}, \tau_{\mathrm{u}}\right)$ We show this as follows :

Let $G_{n}=\left(\frac{-1}{n}, \frac{1}{n}\right), \mathrm{n}=1,2,3, \ldots \ldots G_{n}$ is an open in $\mathrm{R}, \forall \mathrm{n}=1,2,3, \ldots \ldots \ldots$

Now let $G_{n}{ }^{*}=\left(\frac{-1}{n}, \frac{1}{n}\right)^{*}=G_{n} \cap \mathrm{Q}$. Now $\mathrm{Gn} *$ is an open in $\mathrm{Q} \forall \mathrm{n}=1,2,3, \ldots$. But $\bigcap_{n=1}^{\infty} G_{n}{ }^{*}=\{0\}$, which is not pre-open in Q (in fact every singleton is not pre-open in usual topology).

\subsection{Theorem}

Let $\mathrm{X}$ be a pre-A-space, then $\mathrm{MP}(\mathrm{x})$ is a compact set for any $\mathrm{x} \in \mathrm{X}$.

Proof :- Let $\left\{\alpha_{i}:{ }_{i} \in I\right\}$ be an open cover of $\operatorname{MP}(x)$. Then $x$ is in $\alpha_{i}$ for some ${ }_{i} \in I$. Therefore there exist $\mathrm{MP}(\mathrm{x}) \subseteq \alpha \mathrm{i}$. Hence $\alpha \mathrm{i}$ is a finite sub cover of $\left\{\alpha_{\mathrm{i}}:{ }_{\mathrm{i}} \in \mathrm{I}\right\}$. Thus $\operatorname{MP}(\mathrm{x})$ is a compact for any $\mathrm{x} \in \mathrm{X}$.

\subsection{Theorem:-}

Let $\mathrm{X} / \mathrm{d}$ be a quotient space of pre-A-space , then $\mathrm{X} / \mathrm{d}$ is pre-A-space.

Proof :- Let $\mathrm{q}: \mathrm{X} \rightarrow \mathrm{X} / \mathrm{d}$ be a quotient map. Take $\mathrm{A}=\bigcap_{a \in \Lambda} U_{a}$ arbitrary intersection of open set in $\mathrm{X} / \mathrm{d}$, we have

$$
q^{-1}(A)=q^{-1}\left(\bigcap_{a \in \Lambda} U_{a}\right)=\bigcap_{a \in \Lambda} q^{-1}\left(U_{a}\right) \text {. Now } q^{-1}\left(U_{a}\right) \text { is an open set in } X
$$

for any $a \in$, because $q$ is a quotient function. Therefore $q^{-1}(A)$ is a pre - open set in $X$. But $A$ is in $X / d$, hence $\mathrm{X} / \mathrm{d}$ is a pre-A-space.

\subsection{Theorem:-}

Let $\mathrm{X}$ be a pre-A-space, then $\mathrm{X}$ is Hausdoroff if and only if the two conditions are satisfied . 
1) $X$ is a Sub Maximal space.

2) $M P(x) \cap M P(y)=\varnothing$ for any $x \neq y$ in $X$.

Proof :- $\Rightarrow$ ) Let $X$ be Hausdoroff space, then $X$ is $\tau_{1}$-space, hence by Corollary (3.7) $X$ is discrete space , therefore $\mathrm{X}$ is a Sub Maximal space.

Now, we prove the second condition holds.

Let $\mathrm{x}, \mathrm{y} \in \mathrm{X}$ such that $\mathrm{x} \neq \mathrm{y}$, then there exist $\{\mathrm{x}\},\{\mathrm{y}\}$ are two open sets contains $\mathrm{x}, \mathrm{y}$ at respectively, hence $\{\mathrm{x}\},\{\mathrm{y}\}$ are two disjoint minimal pre-open sets contains $\mathrm{x}, \mathrm{y}$ at respectively, because $\mathrm{X}$ is discrete space .

$\Leftarrow)$ Suppose the condition two are satisfied and let $\mathrm{x}, \mathrm{y} \in \mathrm{X}$ such that $x \neq y$, then by second condition , then there exist two minimal pre-open sets $\mathrm{MP}(\mathrm{x})$ and $\mathrm{MP}(\mathrm{y})$ contains $\mathrm{x}$, y at respectively, hence $\mathrm{MP}(\mathrm{x})$ and $\mathrm{MP}(\mathrm{y})$ are pre-open sets contain $\mathrm{x}, \mathrm{y}$ at respectively, but $\mathrm{X}$ is Sub Maximal space, therefore MP(x) and $\mathrm{MP}(\mathrm{y})$ are open sets, by Theorem (2.3). Thus X is Hausdoroff space.

\section{References}

[1] Alexandroff,P. ,Diskrete Raume, Mat. Sb. (N.S.) 2, 501-518. (1937).

[2] Arenas,F.G. ,Alexandroff spaces, Acta Math. Univ. Comenianae , 68 ,17-25(1999).

[3] Arenas F.G., Dontchev J, and Ganster M., On some weaker forms of Alexandroff spaces, Arab. J. Sci. Eng. Sect. A Sci., 23 (1), 79-89,(1998).

[4]Ayed .E. Hashoosh Al-Badry, On Alexandroff spaces with application in computer sciences, M.Sc.Thesis, KufaUniversity,College of Math, and computer sciences,(2011).

[5] Hisham .B. Mahdi and M.S. El-atrash, Characterization of lower separation axioms in $\mathrm{T}_{0}-$ Alexandroff spaces, Proceeding of the First conference on Mathematical Science, Zarqa Private University, Jordan, 77-89 (2006).

[6] Julian, D.,Survey on pre open sets, arXiv: math. GN/ 9810177, Vol. 30, Oct., (1998).

[7] Kovalevsky, V.A., Finite Topology as Applied to Image Analysis ,Computer Vision, Graphic and Image Processing 45, 141-161, (1989).

[8] Mashhour, A. S. M.E. Abd El-Monsef and S.N. El-Deeb,On pre-continuous and weak pre-continuous mappings, Proc. Math. Phys. Soc. Egypt 53 ,47-53,(1982) .

[9] Melin E., Connectedness and continuity in digital spaces with the Khalimsky topology, Uppsala Univeristy, Department of Mathematics, Available at http://www.math.uu.se/research/pub/Melin1.pdf. (2003). [10] Reilly I.L. And Vamanamurthy, M.K.,On some questions concerning pre-open sets, Kyungpook Math. J. 3(1) (1990).

[11] Rosenfeld, A., Connectivity in digital pictures, J. ACM ,17, 146-160. A. 1970.

[12] Rosenfeld, A., Adjacency in digital pictures, Inform, and Control 26, 24-33, 1974.

[13] Veera Kumar, M. K. R. S., Pre semi - closed sets, Indian Journal of Math. Vol. 44, No.2 , 165- 181, (2002). 